\title{
Research on the operational mechanism of network fundraising in Background of "Internet+"
}

\author{
Fu Hongming ${ }^{1, a^{*}}$, Cai Hui ${ }^{2, b}$ and Song Hua \\ ${ }^{1}$ Department of management science and information management, Wuhan University Of \\ Technology, Hongshan District, Wuhan, Hubei, China \\ ${ }^{2}$ Department of Journalism and communication, Wuhan University Of Technology, Hongshan \\ District, Wuhan, Hubei, China \\ ag86009330@whut.edu.cn, b1872020360@qq.com \\ ${ }^{*}$ Corresponding author
}

Keywords: Network fundraising, Theory of planned behavior, Donation intention.

\begin{abstract}
In this study, people of different ages and occupations were selected as the research objects. This study uses the method of questionnaire survey, supplemented by the theory of planned behavior. SPSS19.0 software was used to analyze the results of 500 valid questionnaires. The study found that the attitude of donation, the subjective norm and the perception behavior control factors have positive influence on the public participation in the Internet solicitation. On the basis of this, suggestions are put forward according to the results of data analysis.
\end{abstract}

\section{“互联网+”背景下网络募捐运行机制研究 \\ 富泓茗 ${ }^{1, a^{*}}$, 蔡慧 $2, b$, , 宋华 \\ 1武汉理工大学管理科学与信息管理系, 洪山区, 武汉, 湖北, 中国 \\ 2武汉理工大学新闻传播系, 洪山区, 武汉, 湖北, 中国 \\ a986009330@whut.edu.cn, b1872020360@qq.com \\ 通讯作者}

关键词：网络募捐; 计划行为理论; 捐赠意向

中文摘要.本研究以不同年龄、职业的民众为研究对象, 采用问卷调查的方法, 辅之以计划行 为理论, 运用SPSS19.0软件对 500 份有效问卷结果进行数据分析。研究发现捐赠态度、主观规 范、知觉行为控制因素都对公众参与网络募捐的意向起正面影响作用。在此基础上根据数据 分析结果提出建议。

\section{1. 引言}

随着互联网技术的快速发展, “互联网+” 这种互联网与传统行业相结合的理念渗透到生 活的方方面面。在慈善领域, “互联网+慈善” 的理念主要是以网络募捐形式呈现。相比于 传统的募捐方式, 网络募捐具有传播速度快、受众范围广、影响力度大等优势, 一定程度上 促进了我国公益事业的发展。

然而由于当前网络募捐的法律和监管机制仍处于完善阶段，很多商家钻取法律或监管的 漏洞, 给慈善募捐活动披上 “网络募捐” 的外衣以达到营销目的, 更有甚者借网络募捐之名 行计骗之实。虽然国家出台的《慈善法》中有针对网络募捐的规范条款, 且民政部已经指定 
13 家慈善组织互联网募捐信息平台，但是网络募捐领域仍存在不规范行为，严重损害公众 参与网络募捐的积极性, 阻碍我国公益事业的良性发展。

本文将在《慈善法》和相关法律条文的背景下，从网络募捐平台的运行机制和公众参与 机制两个方面分析网络募捐的运行机制, 并针对网络募捐出现的相应问题提出建设性方案, 以期网络募捐得到良性发展。

\section{2. 文献综述}

依托于迅速发展的互联网技术以及深入社会各个方面“互联网+”模式，网络募捐得以迅 速发展。柯凤华（2008）认为, 网络募捐是通过网络这一媒介, 借助其在一定范围内的辐射 力和影响力, 整合网络资源, 发动网民力量, 为在网络上进行求助的群体提供无偿的经济募 捐的一种非营利行为, 他的研究突出了网络资源整合的特点 ${ }^{[1]}$ 。侯江红等 (2010) 则认为网络 募捐是通过网络, 寻求资金或物质援助, 对募捐做好管理的过程, 重点强调管理过程中的慈 善信息的收集、发布, 善款募捐, 募捐监督, 互动等 ${ }^{[2]}$ 。汪丹 (2014) 等则仅对网络募捐中通 过互联网媒介发布募款或集物信息进行强调 ${ }^{[3]}$ 。

随着近年来 “互联网+慈善” 理念的发展与完善, 尤其是在各种网络募捐平台兴起并广泛 地为个人或慈善机构借助用以发布信息之后, 文献中对网络募捐的研究则普遍强调了募捐平 台的规范性。国家针对此类问题及时出台相关政策条例。2016年颁布的《慈善法》第二十三 条规定，慈善组织通过互联网开展公开募捐的，应当在国务院民政部门统一或者指定的慈善 信息平台发布募捐信息。民政部随后公示首批13家慈善组织互联网募捐信息平台的名单。在 此研究背景下, 邱习强（2017）认为网络募捐是指个人或慈善组织基于某种需求, 通过网络 (含移动网络) 途径, 向不特定对象传递募捐信息, 并利用互联网募捐平台来筹集资金或物 资, 并进行信息反馈的过程 ${ }^{[4]}$ 。汪银辉 (2017) 则就个人求助发起模式和个人与慈善组织合作 发起模式进行分别讨论, 认为后者是一种更为合理的模式 ${ }^{[5]}$ 。徐宇珊 (2017) 对网络募捐反馈 机制进行研究，认为应在网络募捐运行机制当中建立完善的反馈机制[6]。

结合以上观点, 可以得出网络募捐的合理运行机制主要是依托受认可的互联网募捐平台, 由经过审查的慈善组织或个人发起筹资并予以信息反馈。以上研究提到的更多是关于募款方 的内容, 对于捐款方的研究, 也就是对公众网络募捐的参与机制的研究, 见诸文献的并不多。 而研究公众网络募捐参与机制也是对网络募捐运行机制研究中非常重要的一环, 故本研究将 在已有的研究基础之上，对公众网络募捐的参与机制进行研究。

刘秀秀（2014）认为，研究公众网络募捐的参与机制主要还是要从公众的态度和行为入 手 [7]。本研究将根据该思路, 采用计划行为理论, 结合影响公众参与网络募捐意向的因素, 编 制《关于网络募捐大众行为与态度分析调查》调查问卷, 根据得出数据分析出公众参与网络 募捐影响因子。计划行为理论由Ajzen提出, 该理论认为人的行为是经过深思熟虑的计划的结 果 ${ }^{[8]}$ 。大量研究与实践显示, 计划行为理论对人的行为具有良好的解释力与预测力。因此研究 设计将围绕计划行为理论展开, 结合影响因素构建理论研究框架。

\section{3. 研究设计}

计划行为理论认为所有可能影响行为的因素都是经由行为意向来间接影响行为的表现。 而行为意向受到三项相关因素的影响, 其一是源自于个人本身对于采取某项特定行为所持有 的固有 “态度” ; 其二是可能会影响个人采取某项特定行为的“主观规范”; 最后是源自于个人 在采取某项特定行为时所感受到可以控制或掌握的 “知觉行为控制”。依照计划行为理论, 本研究构建公众参与网络捐赠意向理论模型, 如图1所示。 


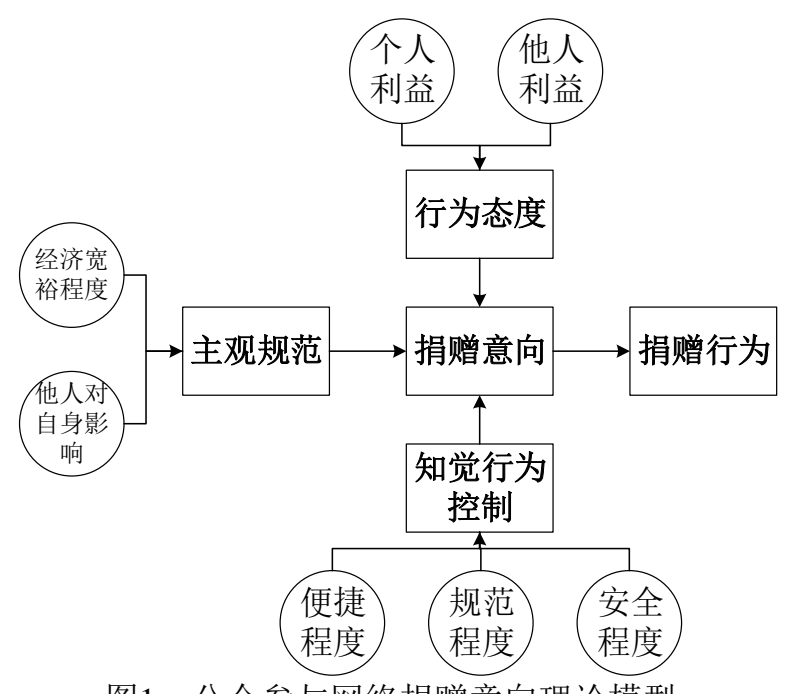

图1 公众参与网络捐赠意向理论模型

由模型可知, 三个影响公众参与捐赠意向的主要方面分别为: 公众对网络捐赠的态度、 公众参与网络捐赠的主观规范以及公众个人知觉行为控制。计划行为理论认为, 在一般情况 下, 行为态度越积极, 主观规范带来的支持度越大, 知觉行为控制感越强, 那么个人做出行 为的意愿就会越强。因此本研究提出如下假设:

$\mathrm{H} 1$ ：捐赠者的捐赠态度对捐赠者本人的捐赠意向有正向作用。

$\mathrm{H} 2$ ：捐赠者的主观规范对捐赠者本人的捐赠意向有正向作用。

$\mathrm{H} 3$ ：捐赠者个人知觉行为控制对捐赠者本人的捐赠意向有正向作用。

根据以上假设以及构建的公众参与网络捐赠意向理论模型，在编制问卷的过程中将三个 影响公众参与捐赠意向主要方面当中的具体因素以及公众的捐赠意向结合实际进行拓展。行 为态度方面因素, 问卷予以 3 个题项进行测量; 主观规范方面因素, 问卷予以 4 个题项进行测 量; 知觉行为控制方面, 问卷予以 6 个题项进行测量; 对于公众的捐赠意向, 问卷予以 2 个题 项进行测量。这 15 个题项均采用李克特五级量表： “ 1 ” 表示不认同, “2” 表示比较不认 可，“3”表示一般，“4”表示比较认同，“5”表示非常认同。

除了以上部分外，问卷还包括 “个人基本信息” 以及 “捐赠信息” 两部分内容。设置其 的主要目的是对样本及其特征进行描述性统计分析。

\section{4. 研究结果分析}

在正式调查之前, 本研究首先对问卷进行预投放, 将问卷按年龄和职业分层发放给参与 过网络募捐的30位捐赠者, 询问其对问卷的整体感受, 以及对一些重要题项的语意是否理 解。结合受测者的反馈与建议, 确定最终问卷。在正式投放过程中, 本研究采取分层随机抽 样的方法进行样本采集, 并利用线上线下并重的方式投放问卷。共投放问卷513份, 其中线 上投放440份, 线下投放 73 份, 有效回收问卷500份, 回收率为 $97 \%$ 。在回收数据之后, 本研 究运用SPSS19.0对数据分别进行描述性统计分析、信度效度检验、因子分析以及回归分析, 并对假设进行检验。

\section{1 描述性统计分析}

首先对样本基本信息进行描述性统计分析，包括性别、年龄、职业、可支配收入四个方 面, 具体情况参见表1。由表1可以看出, 本研究男女比例为47:53, 大体趋近于 $1: 1$, 女性略多 于男性。18-22岁受测者占整体半数左右, 其次占多数的是31-45岁年龄层的受测者。结合职业 和可支配收入水平来看, 18-22岁年龄层受测者大多是在校大学生, 而31-45岁年龄层受测者大 多具有稳定的职业与收入，属于社会的“中生代”群体。 
表1 样本基本特征交叉表

\begin{tabular}{|c|c|c|c|c|c|}
\hline 统计特征 & 成分构成 & \multicolumn{2}{|c|}{ 样本数量 } & 所占比重 $(\%)$ & 合计 \\
\hline \multirow{2}{*}{ 性别 } & 男 & \multicolumn{2}{|c|}{235} & 47.0 & 235 \\
\hline & 女 & \multicolumn{2}{|c|}{265} & 53.0 & 265 \\
\hline \multirow{8}{*}{ 年龄 } & \multirow{2}{*}{ 18-22岁 } & 男 & 97 & 40.9 & \multirow{2}{*}{237} \\
\hline & & 女 & 140 & 59.1 & \\
\hline & \multirow{2}{*}{ 23-30岁 } & 男 & 29 & 58.0 & \multirow{2}{*}{50} \\
\hline & & 女 & 21 & 42.0 & \\
\hline & \multirow{2}{*}{ 31-45岁 } & 男 & 57 & 47.1 & \multirow{2}{*}{121} \\
\hline & & 女 & 64 & 52.9 & \\
\hline & \multirow{2}{*}{ 45岁以上 } & 男 & 52 & 56.5 & \multirow{2}{*}{92} \\
\hline & & 女 & 40 & 43.5 & \\
\hline \multirow{12}{*}{ 职业 } & \multirow{2}{*}{ 公务员 } & 男 & 12 & 54.5 & \multirow{2}{*}{22} \\
\hline & & 女 & 10 & 45.5 & \\
\hline & \multirow{2}{*}{ 企业单位管理 } & 男 & 44 & 71.0 & \multirow{2}{*}{62} \\
\hline & & 女 & 18 & 29.0 & \\
\hline & \multirow{2}{*}{ 教师 } & 男 & 32 & 37.2 & \multirow{2}{*}{86} \\
\hline & & 女 & 54 & 62.8 & \\
\hline & \multirow{2}{*}{ 自由职业者 } & 男 & 23 & 46.9 & \multirow{2}{*}{49} \\
\hline & & 女 & 26 & 53.1 & \\
\hline & \multirow{2}{*}{ 学生 } & 男 & 98 & 42.8 & \multirow{2}{*}{229} \\
\hline & & 女 & 131 & 57.2 & \\
\hline & \multirow{2}{*}{ 其他 } & 男 & 26 & 50.0 & \multirow{2}{*}{52} \\
\hline & & 女 & 26 & 50.0 & \\
\hline \multirow{10}{*}{ 可支配收入 } & \multirow{2}{*}{$0-2000$ 元 } & 男 & 92 & 41.3 & \multirow{2}{*}{223} \\
\hline & & 女 & 131 & 58.7 & \\
\hline & \multirow{2}{*}{ 2001-4000元 } & 男 & 46 & 42.2 & 109 \\
\hline & & 女 & 63 & 57.8 & 109 \\
\hline & $4001-6000=$ & 男 & 41 & 55.4 & 74 \\
\hline & 4001-000U兀 & 女 & 33 & 44.6 & 14 \\
\hline & & 男 & 20 & 46.5 & 13 \\
\hline & 6001-8000兀 & 女 & 23 & 53.5 & 43 \\
\hline & 8000 元以上 & 男 & 36 & 70.6 & 51 \\
\hline & 0000ルレ上 & 女 & 15 & 29.4 & כ1 \\
\hline
\end{tabular}

在对 “捐赠态度”、“主观规范”、“知觉行为控制”、“捐赠意向” 各个变量进行描述 性统计分析中 (结果参见表2) 可以发现, 各变量均值大部分在3以上; 行为态度方面变量当 中 “参与网络募捐会使自己心里得到安慰并感到高兴” 问项均值最高; 主观规范方面变量当 中 “自己喜欢的公众人物的呼吁” 问项均值为 2.48 , 低于 3 ; 知觉行为控制方面变量均值普遍 偏高; 而受测者捐赠意向两个题项的均值处于 3 和 3.5 之间。

表2 变量描述性统计分析结果

\begin{tabular}{|c|c|c|c|c|c|c|}
\hline \multirow{2}{*}{ 变量 } & \multirow{2}{*}{ 问卷项目 } & \multirow{2}{*}{ 统计量N } & \multicolumn{2}{|c|}{ 均值 } & \multirow{2}{*}{\begin{tabular}{|c|} 
标准差统 \\
计量 \\
\end{tabular}} & \multirow{2}{*}{\begin{tabular}{|c|} 
方差统计 \\
量 \\
\end{tabular}} \\
\hline & & & 统计量 & 标准误 & & \\
\hline \multirow{3}{*}{ 捐赠态度 } & X101 参与网络募捐会使自己心里得到安慰并感到高兴 & 500 & 4.08 & .041 & .916 & .840 \\
\hline & X102 网络募捐能够帮助真正有困难的人解决难题 & 500 & 3.62 & .040 & .890 & .792 \\
\hline & X103 参与网络募捐能够提升自我价值 & 500 & 3.36 & .043 & .955 & .912 \\
\hline \multirow{4}{*}{ 主观规范 } & X201 经济宽裕 & 500 & 3.74 & .051 & 1.135 & 1.289 \\
\hline & X202 家人的支持 & 500 & 3.85 & .048 & 1.068 & 1.140 \\
\hline & X203 好友在社交软件转发的捐赠信息 & 500 & 3.37 & .050 & 1.119 & 1.252 \\
\hline & X204 自己喜欢的公众人物的呼吁 & 500 & 2.48 & .057 & 1.279 & 1.637 \\
\hline \multirow{6}{*}{$\begin{array}{c}\text { 知觉行为控 } \\
\text { 制 }\end{array}$} & X311 我认为现在大家普遍选择网络募捐平台是因为筹款比较便捷, 易于传播 & 500 & 3.73 & .048 & 1.083 & 1.173 \\
\hline & X312 捐赠方式流程需要简洁化 & 500 & 3.72 & .044 & .994 & .988 \\
\hline & X321 募捐人信息亟待透明化 & 500 & 3.94 & .055 & 1.241 & 1.539 \\
\hline & X322 网络募捐需要保证本人信息安全和隐私 & 500 & 4.06 & .046 & 1.036 & 1.074 \\
\hline & $\begin{array}{l}\text { X331 现在网络募捐的平台软件（如轻松筹、腾讯公益）太多，但是缺少一个 } \\
\text { 由政府审核资料、认可担责的网络募捐平台 }\end{array}$ & 500 & 4.14 & .048 & 1.070 & 1.144 \\
\hline & X332 我认为如今急需出台有关网络募捐的法律条例 & 500 & 4.23 & .045 & 1.003 & 1.005 \\
\hline \multirow{2}{*}{ 捐赠意向 } & Y1 今后在各平台发现网络募捐信息我会选择参与捐助 & 500 & 3.37 & .036 & .799 & .638 \\
\hline & Y2 今后在各平台发现网络募捐信息我会转发给身边的人 & 500 & 3.22 & .034 & .768 & .590 \\
\hline
\end{tabular}

\section{2 信度效度检验}


在对数据进行分析之前, 需要进行信度检验, 主要采取克朗巴哈系数进行测量。结果如 表 3 所示, 样本的得克朗巴哈系数为 0.807 , 大于 0.7 ; 各变量的克朗巴哈系数分别为 $0.618,0.680$, $0.714,0.907$, 均大于 0.7 , 所以认为此次调查问卷可信度较高。

表3 统计量Cronbach a 值

\begin{tabular}{|c|c|}
\hline 变量 & Cronbach \\
\hline 捐赠态度 & 0.618 \\
\hline 主观规范 & 0.680 \\
\hline 知觉行为控制 & 0.714 \\
\hline 捐赠意向 & 0.907 \\
\hline
\end{tabular}

利用KMO检验与Barrlett's球形检验对数据进行效度分析, 判断变量是否适合做因子分析。 结果如表4所示, 显示 $\mathrm{KMO}$ 检验值为 0.821 , Bartlett's球形检验 $\mathrm{P}$ 值为 $0.000<0.05$, 适合做因子分 析。

表4 各个量表KMO和Bartlett球形检验结果

\begin{tabular}{|c|c|c|c|}
\hline & 捐赠态度 & 主观规范 & 知觉行为控制 \\
\hline $\mathrm{KMO}$ 检验 & 0.919 & 0.712 & 0.706 \\
\hline Bartlett & 0.000 & 0.000 & 0.000 \\
\hline
\end{tabular}

\section{3 因子分析}

按照主成分分析法提取因子,可提取 3 个主因子,累计贡献率为 $92.952 \%$,即可以反映原始 15 个变量 $92.952 \%$ 的信息量。影响因素的特征根和贡献率参见表 5 。

表5 影响因素的特征根和贡献率

\begin{tabular}{|c|c|c|c|}
\hline 主因子 & 捐赠态度 & 主观规范 & 知觉行为控制 \\
\hline 特征根 & 1.754 & 1.079 & 1.885 \\
\hline 贡献率(\%) & 43.860 & 26.971 & 22.121 \\
\hline 累计贡献率(\%) & 43.860 & 70.831 & 92.952 \\
\hline
\end{tabular}

对 3 个主因子进行方差最大化正交旋转,得到因子载荷矩阵。旋转因子载荷矩阵如表6所 示，由表6可以看出第一主成分与X101、X102、X103具有较大载荷; 第二主成分与X201、 X202、X203、X204具有较大载荷; 第三主成分与X311、X312、X321、X322、X331、X332 具有较大载荷。

表6 影响因素正交旋转因子载荷矩阵

\begin{tabular}{|c|c|c|c|}
\hline & \multicolumn{3}{|c|}{ 主因子 } \\
\hline & 1 & 2 & 3 \\
\hline X101 & .715 & .089 & -.036 \\
\hline X102 & .839 & .075 & -.015 \\
\hline X103 & .703 & -.114 & .099 \\
\hline X201 & .040 & .698 & .249 \\
\hline X202 & .006 & .874 & .053 \\
\hline X203 & -.023 & .525 & .347 \\
\hline X204 & -.038 & .580 & -.042 \\
\hline X311 & .010. & .085 & .502 \\
\hline X312 & .041 & .367 & .710 \\
\hline X321 & .058 & .382 & .586 \\
\hline X322 & .094 & .059 & .498 \\
\hline X331 & .001 & .136 & 811 \\
\hline X332 & .050 & .223 & 843 \\
\hline
\end{tabular}




\section{4 相关分析}

从表7的相关系数可以看出, 捐赠意向与捐赠态度、主观规范及知觉行为控制均呈正向相 关, 其中与主观规范的相关性最高, 然后依次是知觉行为控制和捐赠态度。为了进一步检验 提出的假设，本文将采用回归分析加以证明。

\begin{tabular}{|c|c|c|c|c|}
\hline & 1 & 2 & 3 & 4 \\
\hline 1 捐赠态度 & 1 & & & \\
\hline 2 主观规范 & .041 & 1 & & \\
\hline $\begin{array}{c}3 \text { 知觉行为 } \\
\text { 控制 }\end{array}$ & .077 & .716 & 1 & \\
\hline 4 捐赠意向 & .080 & .132 & .088 & 1 \\
\hline
\end{tabular}

\section{5 回归分析}

为检验本文提出的三个假设，了解公众对网络捐赠的态度、促进或阻碍公众参与网络捐 赠的主观规范以及公众个人知觉行为控制对公众参与网络捐赠意向的影响程度, 分别以捐赠 态度 $(X 1)$ 、主观规范 $(X 2)$ 、知觉行为控制 $(X 3)$ 为自变量, 以捐赠意向 $(Y 1)$ 为因变量, 探讨它们的关系。结果如表 8 , 表 9 , 表10所示。

根据表 8 的结果, 受测者行为态度与参与捐赠意向的回归方程为:

$$
Y=3.614+0.087 X 1
$$

回归方程的系数是 0.087 为正数, 说明受测者行为态度对参与捐赠意向有正向影响。本文 假设H1成立。

表8 捐赠态度与捐赠意向的回归分析结果

\begin{tabular}{|c|c|c|c|c|c|c|c|}
\hline \multirow{2}{*}{ 模型 } & \multicolumn{2}{|c|}{ 非标准化系数 } & 标准系数 & \multirow{2}{*}{$\mathrm{t}$} & \multirow{2}{*}{ Sig. } & \multirow{2}{*}{$\mathrm{R}$ 平方 } & \multirow{2}{*}{ 调整R平方 } \\
\hline & $\mathrm{B}$ & 标准误差 & 试用版 & & & & \\
\hline (常量) & 3.614 & .181 & & 19.963 & .000 & \multirow{2}{*}{.006} & \multirow{2}{*}{.004} \\
\hline 捐赠态度 & .087 & .048 & .080 & 1.794 & .073 & & \\
\hline
\end{tabular}

根据表 9 的结果, 受测者主观规范与参与捐赠意向的回归方程为:

$$
Y=2.855+0.131 X 2
$$

回归方程的系数是 0.131 为正数, 说明受测者主观规范对参与捐赠意向有正向影响。本 文假设H2成立。

表9 主观规范与捐赠意向的回归分析结果

\begin{tabular}{|c|c|c|c|c|c|c|c|}
\hline \multirow{2}{*}{ 模型 } & \multicolumn{2}{|c|}{ 非标准化系数 } & 标准系数 & \multirow{2}{*}{$\mathrm{t}$} & \multirow{2}{*}{ Sig. } & \multirow{2}{*}{ R平方 } & \multirow{2}{*}{ 调整R平方 } \\
\cline { 2 - 6 } & $\mathrm{B}$ & 标准误差 & 试用版 & & & \\
\hline (常量) & 2.855 & .151 & & 18.862 & .000 & \multirow{2}{*}{.018} & \multirow{2}{*}{.016} \\
\cline { 2 - 6 } 主观规范 & .131 & .044 & .132 & 2.982 & .003 & \\
\hline
\end{tabular}

根据表10的结果, 受测者知觉行为控制与参与捐赠意向的回归方程为:

$$
Y=2.972+0.081 X 3
$$

回归方程的系数是 0.081 为正数, 说明受测者知觉行为控制对参与捐赠意向有正向影

\begin{tabular}{|c|c|c|c|c|c|c|c|}
\hline \multirow{2}{*}{ 模型 } & \multicolumn{2}{|c|}{ 非标准化系数 } & 标准系数 & \multirow[b]{2}{*}{$\mathrm{t}$} & \multirow{2}{*}{ Sig. } & \multirow{2}{*}{$R$ 平方 } & \multirow{2}{*}{ 调整 $\mathrm{R}$ 平方 } \\
\hline & $\mathrm{B}$ & 标准误差 & 试用版 & & & & \\
\hline (常量) & 2.972 & .166 & & 17.849 & .000 & \multirow[b]{2}{*}{.008} & \multirow[b]{2}{*}{.006} \\
\hline $\begin{array}{c}\text { 知觉行为 } \\
\text { 控制 }\end{array}$ & .081 & .041 & .088 & 1.982 & .048 & & \\
\hline
\end{tabular}
响。本文假设H3成立。

表10 知觉行为控制与捐赠意向的回归分析结果

\section{5. 结果讨论}


本研究从公众参与网络募捐的行为着手, 运用计划行为理论, 研究公众网络募捐的参与 机制, 通过分析捐赠态度、主观规范和知觉行为控制与捐赠意向的相关关系, 得出结论: 捐 赠态度、主观规范、知觉行为控制因素都对公众参与网络募捐的意向起正面影响作用。

具体来看，18-22岁年龄层受测者大多是在校大学生，对于诸如网络募捐一类新兴的 “互 联网+”产物接受频次最多; 而31-45岁年龄层受测者大多具有稳定的职业与收入，对于事物 有较高的感知能力。受测者对于问卷题项的因素考量有着一定程度的认可且受测者的测试结 果相互之间存在较大差异。

研究还显示公众人物的呼吁并不会对公众参与捐赠有显著的影响, 这也说明信息时代公 众对于信息的选择有一定的侧重性, 频繁的公众人物信任危机事件可能是诱发这一结果的主 因。结合相关分析与回归分析, 可以得知公众对于网络募捐态度越积极, 参与网络募捐的意 向就越大, 且公众的经济情况以及身边其他人会影响到公众自身捐赠的意向。另外知觉行为 控制中的便捷程度、规范程度以及安全程度因素对于公众参与网络募捐意愿具有一定的预测 能力, 说明随着公众法律意识、自我保护意识和先进理念认识的不断提高, 网络募捐需要从 各个方面提高水平, 以满足公众需求。

根据研究成果及结论, 拟提出以下建议:

1) 完善网络募捐的反馈机制，在保护好捐赠者个人信息安全的前提下，使捐赠者接受网 络募捐项目进度反馈。

2）面向公众公布网络募捐项目内容及成果，在公众面前树立良好形象。

3）募捐平台应注重捐赠者的用户体验，简化捐赠流程，并保证捐赠者的信息安全。

4）针对捐赠者权益的保护出台更精细的监管机制，切实保护捐赠者的利益。

本研究的不足之处在于对公众网络募捐参与机制研究仅仅局限于对参与过捐赠的人进行 调查研究, 没有考虑对网络募捐感兴趣却还没有参与捐赠的人群。后续研究中可以加以考虑。

\section{致谢}

本文为武汉理工大学自主创新研究基金本科生项目《“互联网+”背景下的网络募捐的运 行机理和发展模式研究》阶段性成果之一。

\section{References}

[1] Ke Fenghua, Research on Normalization of On-line Solicitation[D]. Jinan University, 2008.

[2] Hou Hongjiang, Xu Mingxiang and Zhang Kankan, Research on the model of non profit organization fund-raising based on Network[J], JOURNAL OF SICHUAN ADMINISTRATION COLLEGE, 2010,(6):86-89. DOI:10.3969/j.issn.1008-6323.2010.06.021.

[3] Wang Dan, Research on the sustainable development of China's network charity[J], Journal of Social Work, 2014,(6):91-98. DOI:10.3969/j.issn.1672-4828.2014.06.012.

[4] Qiu Xiqiang, Research on the Comprehensive Supervision Mechanism of China's Internet Fundrasing[D]. Shandong University, 2017.

[5] Wang Yinhui, On the joint liability of Network fundraising Platform[D]. Gansu Political Science And Law Institute, 2017.

[6] Xu Yushan, Improve the Feedback Mechanism of Charitable Organizations' Fundraising[J], China Opening Journal, 2017(03):89-92.

[7] Liu Xiuxiu, Mobilization and participation: A Study on the donation mechanism of network charity[J], Fujian Tribune, 2014(01):187-192. 
[8] Ajzen, The Theory of Planned Behavior[J], Organizational Behavior and Human Decision Process, 1991, (50):179-211. 